\title{
Un campo di papaveri
}

\author{
Andreas F. Kelletat
}

Gern hatte Sottkowski die Einladung zu der Athener Germanistentagung über Erinnerung und Identität angenommen und war schon ein paar Tage vor Konferenzbeginn nach Griechenland geflogen. Er musste einfach mal raus aus der Tretmühle seiner Gommersbacher Hochschule. Im Hotel Orion gleich am Strefi-Park hatte er sich ein Zimmer besorgt, oben am Rand des quirlig gemütlichen Anarchistenviertels Exarchia. Von seinem Balkon aus konnte er über die ganze Stadt schauen, bis zur Akropolis und zum Saronischen Golf und den Gebirgszügen im Westen, hinter denen abends die Sonne unterging. Die Tage nutzte Sottkowski für ausgedehnte Spaziergänge und für Museumsbesuche. Er schaute sich wie schon 1978 während seiner ersten Griechenlandreise das Archäologische Nationalmuseum an und stand jetzt ein bisschen wehmütig vor den Kouroi, den kraftstrotzenden Jünglingsstatuen. Er ging ins Benaki-Museum für Kykladische Kunst, ins Schliemann-Haus und auch ins Byzantinische Museum, wo sie seine Lieblingsikone aus dem Depot geholt und ausgestellt hatten, die mit dem hundsköpfigen Christophorus, den Kynokephalos aus dem 17. Jahrhundert. Die dort seit Herbst ebenfalls gezeigte Andy-Warhol-Ausstellung war leider schon vorbei. Gerne hätte Sottkowski gesehen, ob und wie sich dessen Selbstporträts und dessen Pop-Bilder von Marilyn Monroe, Mao, Elvis Presley oder Joseph Beuys neben den altfrommen Heiligenbildern behaupten können. Am letzten freien Tag, einem Mittwoch, hatte er sich den Kerameikos am anderen Ende der Innenstadt vorgenommen, das von Touristen so erfreulich verschonte Ausgrabungsgelände unweit der Akropolis.

Die beiden Burschen in ihren schwarz-rot gestreiften Kapuzenpullovern waren Sottkowski gleich aufgefallen. Waagrecht angeordnet waren die breiten Streifen bei dem einen, senkrecht bei dem anderen. Sie standen vor dem schmiedeeisernen Tor der Synagoge in der Melidonistraße. Sein Griechisch reichte, um die Inschrift unter dem Sims des Gebäudes zu entziffern: Synagoge der israelitischen Gemeinschaft zu Athen. Dann war da noch so etwas wie Beth Shalom zu lesen. Der waagrechte Kapuzenbursche fotografierte den senkrechten und anscheinend sollte der sechszackige Stern mit aufs Bild kommen. Der eine hielt in der rechten Hand die Kamera und gab mit der linken Zeichen, dass sein Kumpel mit dem Kopf noch ein bisschen dichter an das Gitter mit dem Davidstern heranrücken müsse. Der streckte seinen rechten Arm nach vorne und spreizte Zeige- und Mittelfinger zum Victory-Zeichen. Sie lachten. 
Im Vorübergehen schaute Sottkowski sich das an. Er blieb sogar kurz stehen. Aber er traute sich dort nicht, ein Foto von den beiden zu machen. Anfang zwanzig dürften sie gewesen sein. Er fand, dass sie trotzdem gut aussahen in ihren gestreiften Kapuzenpullovern. Sie waren schlank, trugen Springerstiefel und hatten kahl rasierte Schädel, Glatzen. Erst als er an der Synagoge vorbei war und oben an der Ecke zur Hermesstraße durch den Zaun schon auf das tiefer liegende Ausgrabungsgelände schauen konnte, knipste er die beiden von weitem dann doch.

Bevor er den Eingang zum Kerameikos-Museum erreicht hatte, drehte er sich noch einmal um. Am Ende der leicht ansteigenden Straße, in vielleicht zwei Kilometer Entfernung, war trotz des trüben Wetters das Parlamentsgebäude am Syntagmaplatz zu erkennen, König Ottos bavarischer Prachtbau. Wie eine vergrößerte Version der Villa Ludwigshöhe kam ihm das vor, wohin er von Gommersbach aus gelegentlich fuhr, um im Pfälzer Wald zu wandern. Links vom Schloss war der Lykavittos zu erkennen, Athens höchster Berg mit der kleinen Georgs-Kapelle und der riesigen griechischen Fahne. Rechts neben der Straße läuft die Bahnlinie und dann kommt die Akropolis. Direkt an der Bahn übernachteten Leute, Afrikaner, in Behausungen aus Pappkartons und Plastikplanen, ein richtiges kleines Dorf. Als er diese Stelle auf einem Spaziergang entdeckt hatte, gleich am ersten Tag, war es noch kälter gewesen als heute, und er hatte auch ein bisschen Angst gehabt vor den Schwarzen, die ihn aus ihren Schlafsäcken und Wolldecken anstarrten wie einen Fremden.

Am Eingang, neben dem Häuschen mit der Museumskasse, stand auf einem handgemalten Zettel: The WC is not working. Der griechische Text war dreimal so lang. Aber das Klo ließ sich zum Glück dennoch benutzen. Kurz nach ihm kamen auch die Kapuzenjungs ins Museum. Gleich fingen sie wieder mit dem Fotografieren an, obwohl die Aufseherinnen immerzu riefen No flash! No flash! Doch das schien die beiden nicht zu kümmern und irgendwann hörten die Frauen auf und ließen sie einfach machen.

In der Eingangshalle schaute Sottkowski sich den Löwen an mit den kreisrunden Augen und der wunderschönen Mähne, der von einer deutschen Archäologin unlängst entdeckt worden sein soll. Seit 1913 graben die Deutschen jetzt schon hier, las er auf der Tafel neben dem Löwen. Die Jungs müssen Italiener sein, dachte er. Vor der großen Statue des nackten Jünglings redeten sie vom Scultore Michelangelo, von Perfetta muscolatura del torso. Sie machten sich gegenseitig auf das geflochtene Haar aufmerksam, das dem Kouros bis zu den Schulterblättern reicht, und zeigten auf die abgebrochene Nase und das leicht lädierte Gemächt. Dann betatschten sie die Pobacken des Jünglings. Eine Aufseherin kam angerannt. Don't touch! rief sie, Don't touch! - Va bene, Va bene sagten die beiden und hoben die Arme in die Höhe, als wollte die Frau sie erschießen.

Im Lichthof machten sie sich über den Stier lustig, knipsten dessen gewaltige Hoden von vorn und von hinten. Red bull, Bull shit, sagte der eine und dann hörte man so etwas wie Red Skin und Fuck parade, aber Sottkowski war sich nicht sicher. Der mit den senkrechten Streifen erklärte seinem Kumpel richtig sachlich, aber ziemlich lautstark etwas von Mitologia greca, von Grande Madre 
und Orgastica, von Kybele und Attis. Mehrmals fiel das Wort Castrazione. Dabei lachten sie und schauten durch die Beine des Stiers zu ihm hinüber.

Als er schon eine Weile vor den Vitrinen mit den Keramiksachen gestanden hatte und ihn die Jungs auf ihrem Rundgang endlich eingeholt hatten, stieß ihm der eine plötzlich mit dem Ellbogen in die Seite und zeigte auf ein kleines rötliches Tongefäß. Er erklärte etwas auf Italienisch, aber Sottkowski verstand das nicht. Nur die Wörter Svastica und Emblema nazista konnte er aus dem Wortschwall heraushören. So etwas war wirklich auf dem Ausstellungsstück zu sehen, neben zwei Kriegern, die mit Lanzen um ein zwischen ihnen stehendes Pferd zu streiten schienen. Wie Strichmännchen kamen ihm die Gestalten vor. Individual Find (800-775 BC) - warriors taming a horse. This is one of the earliest depiction of human figurine, stand auf einem Schildchen am Rand der Vitrine. Si, si, svastica, but not the emblema nazista, kauderwelschte Sottkowski zu dem Burschen und versuchte, ihm dabei in die Augen zu blicken.

Dann ging er in den nächsten Raum, um sich weitere Grabungsfunde anzuschauen, während die beiden wieder ihre Fotos machten. No flash! No flash!, äfften sie die Frauen nach. Red bull, Bull shit! Fuck parade! Schon wieder lachten sie. Auch in diesem Raum entdeckte er jetzt unter den vielen Objekten eine Tonschale mit Hakenkreuzen. Zwischen einer Reihe von Tieren waren sie rund an die Innenwand des kleinen rötlichen Gefäßes gemalt, einer Trinkschale vielleicht, mit schwarzer Tusche wie von Kinderhand. Was für Tiere das sein sollten, war nicht richtig zu erkennen, ob Esel oder Pferde oder Kühe oder doch eher Ziegen, vielleicht sogar Windhunde. Das Schild mit der englischen Erläuterung fehlte bei diesem Ausstellungsstück mit der Nummer 18.

Er ging vor die Tür und steckte sich eine Gauloise an. Liberté toujours stand auf der Packung und dass Nikotin impotent mache. Er schlenderte durch das Museumsgelände. Quer über die Wiese war durch das hoch gewachsene Gras ein Weg gemäht. Es roch nach fauligem Heu. An der Stadtmauer wuchsen Brennnesseln. City Wall stand auf einem der Steine. Xerxes, Salamis, Themistokles, Perikles - all der Schutt aus seiner Schulzeit ging Sottkowski durch den Kopf und er schaute durch den Zaun an der Hermesstraße hinauf zur Akropolis. Da soll die Hakenkreuzfahne geweht haben, hatte ihm sein Vater vor vielen Jahren erzählt. Ein irrer Grieche habe die Fahne eines Nachts runtergeholt, einfach geklaut, gleich Ende Mai 1941. Heilfroh sei er gewesen, dass er damals nicht zu den Wacheschiebenden gehört habe, in jener mondbeglänzten Zaubernacht da oben bei den erhabenen antiken Tempeln.

Am Bach, der durch das Gelände fließt, hielt Sottkowski Ausschau nach den berühmten Riesenschildkröten. Aber er konnte keine entdecken. Vielleicht war es denen noch zu kalt oder sie hielten sich tagsüber versteckt. Auch dort roch es ein bisschen nach Verfaultem. Aus einer gelben Plastikröhre floss klares Wasser in den mit Pflanzen überwucherten Bach. Biotop, Soziotop, Genotop, Nekrotop, murmelte er vor sich hin. Dann schaute er zur Kirche Hagia Triada und zu den Palmen, die ihr bis übers Dach reichen. Was könnte man da noch alles entdecken, dachte er, wenn sie unter der Kirche auch mal graben würden. 
Am Ende des Geländes, wo hinter dem Zaun die Straße nach Piräus entlangläuft, wo sich das Gazi-Viertel rund um das alte Gaswerk zu einem Vergnügungs- und Kulturzentrum mausert und wo Riesenneubauten hochgezogen werden, blieb er vor einem kleinen mit Olivenbäumen und Zypressen umgebenen Hügel stehen. Zwischen den Grabstelen roch es nach Minze. Am Stamm einer Kiefer lehnte ein Handfeuerlöscher. Er knipste Fotos von dem nicht mehr richtig funktionstüchtig aussehenden roten Gerät, von den gelben Blüten des Löwenzahns und von den weißen der Margeriten. Zypressen, meine dunklen Lieblingsbäume, / Ihr werdet stets in meinen Lichtvisionen stehn. Muss dir der Däubler gerade hier und gerade jetzt einfallen?, dachte er.

Dann waren die beiden Kapuzenjungs schon wieder zu hören. Sie kamen oben vom Museum durch das Stelenfeld angelaufen und winkten ihm, dass er $\mathrm{zu}$ ihnen kommen solle. In der Mitte des Hangs waren sie vor einem eingesackten Grab stehen geblieben, verwildert sah es aus mit dem sprießenden Unkraut. Zwischen dem Gras und dem Löwenzahn wuchs überall Klatschmohn. Die Jungs zeigten auf die leuchtend roten Blätter und die schwarzen Punkte im Inneren der Blüten. Sie beugten sich zur Erde, als wollten sie an ihnen schnuppern. Wie auf Verabredung riefen sie gleichzeitig und völlig aufgekratzt: Un campo di papaveri! Un campo di papaveri!

Sie baten Sottkowski ein Foto $\mathrm{zu}$ machen, von diesem Grab mit den Mohnblüten. Sie setzten sich auf eine umgestürzte Stele und machten ihm durch Gesten klar, dass die Papaveri, die Mohnblüten unbedingt mit aufs Bild kommen müssten. Der eine legte seinen Arm um die Schulter des anderen. Der stülpte sich die Kapuze über, streckte im Sitzen seinen rechten Arm nach vorne und machte wieder dieses V-Zeichen. Grazie, mille grazie sagten sie, als er sie geknipst hatte. Sie standen auf und nahmen ihm den Fotoapparat wieder ab.

Er fragte sie auf Englisch, warum sie das mit dem Nazi-Gruß immer noch machen müssten. Sie grinsten, fielen sich um den Hals und küssten sich auf den Mund. Dabei schauten sie ihm in die Augen. Fuck parade! - Kolossal romantisch, was? hörte er den einen, den senkrecht gestreiften, plötzlich auf Deutsch sagen. Was seid ihr bloß für miese Typen, schrie Sottkowski. Aber die beiden lachten nur und der waagrecht gestreifte richtete die Kamera auf ihn. Wo bin ich hier eigentlich, rief er, drehte sich um und hetzte quer über das Mohnblütenfeld den Grabhügel hinauf, zurück zum Ausgang des Museums. 